\title{
On Specifying Real-Time Systems in a Causality-Based Setting ${ }^{\star}$
}

\author{
Joost-Pieter Katoen $^{a}{ }^{\star \star}$, Rom Langerak $^{a}$, Diego Latella ${ }^{b}$ and Ed Brinksma ${ }^{a}$ \\ ${ }^{a}$ Faculty of Computing Science, University of Twente \\ P.O. Box 217, 7500 AE Enschede, The Netherlands \\ ${ }^{b}$ CNUCE Istituto del CNR, Via Santa Maria 36, 56100 Pisa, Italy
}

\begin{abstract}
Event structures are a prominent noninterleaving model for concurrency. Real-time event structures associate a set of time instants to events, modelling absolute time constraints, and to causal dependencies, modelling relative delays between causally dependent events. We introduce this novel temporal model and show how it can be used to provide a denotational semantics to a real-time variant of a process algebra akin to LOTOS. This formalism includes a timed-action prefix which constrains the occurrence time of actions, a timeout and watchdog (i.e., timed interrupt) operator. An event-based operational semantics for this formalism is presented that is shown to be consistent with the denotational semantics. As an example we use an infinite buffer with time constraints on the message latency and the rates of accepting and producing data.
\end{abstract}

\section{Introduction}

Timed extensions of interleaving models for concurrency have been investigated thoroughly in the last decade. Although there are many different ways in which time can be incorporated in labelled transition systems, the most prominent interleaving model, it seems that this issue is quite well-understood, cf. the recipes and proposals in [3] and [21].

Interleaving models are appropriate for the description of the system's observational behaviour. There, it suffices to consider the system as a black box, i.e., not taking into account that a system is composed of subsystems. This applies, for instance, to the field of conformance testing where usually (and often deliberately) no knowledge is available about the internal structure of the system. Also in the final realization phase when (part of) a specification needs to be implemented on a single processor, interleaving models suffice.

\footnotetext{
* The work in this paper is partially funded by C.N.R. - Progetto Bilaterale: Estensioni probabilistiche e temporali dell'algebra di processi LOTOS basate su strutture di eventi, per la specifica e analisi quantitative di sistemi distribuiti, by C.N.R. - Progetto Coordinato: Strumenti per la specifica e verifica di proprieta' critiche di sistemi concorrenti e distribuiti, and by the EU as part of the ESPRIT BRA project 6021: Building Correct Reactive Systems (REACT).

${ }^{\star \star}$ Correspondence to: J.-P. Katoen, e-mail: katoen@cs.utwente.nl, fax: +31-53-4893247.
} 
Interleaving models are not that appropriate for design stages in which the distribution aspects of the system play a prominent rôle. The global state assumption of interleaving models hampers to faithfully model that a system consists of several co-operating subsystems at different locations, each having its own local state. In this design stage the system is considered as a white box where the internal system structure prevails. In particular, if the specification serves as a prescription for the system's implementation rather than as a reference for the observational behaviour of a system, interleaving models become unattractive or even misleading since the independence of actions is not reflected properly [28]. Also for an important design technique, known as action refinement, where an abstract action is implemented by a concrete behaviour, it appears that noninterleaving models are more appropriate, see, e.g., [26].

The incorporation of quantitative information in noninterleaving models, such as event structures [29], pomsets [24], and Mazurkiewicz traces [19], has received scant attention in the literature. Since these models seem to be attractive at the design stages in which the observational behaviour is no longer prevalent, but where the intensional system characteristics dominate, one might even argue that such models in particular should deal with issues like time and probability. In these design stages it is of utmost importance how actions are scheduled in time and with what probability certain alternative executions, which at a more high level of abstraction could be faithfully modelled by means of nondeterminism, can appear.

This paper therefore proposes a real-time extension of (a variant of) event structures; probabilities are dealt with in $[7,15]$. The real-time model is used as a vehicle to provide a denotational semantics to a temporal process algebra based on a kernel which is akin to LOTOS [5]. This formalism includes a timed action-prefix operator which constraints the occurrence time of actions, and a timeout and watchdog (i.e., timed interrupt) operator.

The inclusion of time in partial-order models is not new: e.g., extensions are known of pomsets [8], configurations [18], \{and, or \}-automata [12], sets of posets [13] and event structures [20]. The timed extension of causal trees [10] resembles our model. We are, however, unaware of any proposal that incorporates time, timeouts, and watchdogs in a partial-order setting. These ingredients are considered to be essential to specify real-time systems.

We use Langerak's extended bundle event structures [17], an adaptation of Winskel's event structures [29] to fit the specific requirements of multi-party synchronization and disruption $([>)$. Since we believe that both interleaving and noninterleaving models are legitimate and complementary in the system design process we also consider an event-based operational semantics for the real-time process algebra at hand which, by omitting event identifiers, results in an interleaving semantics. The two semantics are proven to coincide (i.e., strong timed (event) bisimulation equivalent) and thus can be used in a coherent way. This also facilitates the comparison of our timed partial order model and the wealth of existing timed interleaving models. ${ }^{3}$

\footnotetext{
${ }^{3}$ For space reasons the proofs are omitted from this paper; they can be found in [14].
} 


\section{The language}

This paper is based on the process algebraic language PA, in fact LOTOS with a somewhat more concise syntax, generated by the following grammar:

$$
B::=\mathbf{0}|\sqrt{ }| a ; B|B+B| B \|_{G} B|B[H]| B \backslash G|B>>B| B[>B \mid P .
$$

We assume a given set of observable actions Act and an additional invisible action $\tau ; \tau \notin$ Act. The special action $\delta$, which is not user-definable, indicates the successful termination of a behaviour; $\delta \notin$ Act. 0 denotes inaction; $\sqrt{ }$ represents the successful termination of a behaviour. $a$; $B$ denotes the action-prefix of $a \in \operatorname{Act} \cup\{\tau\}$ and $B$. The choice between $B_{1}$ and $B_{2}$ is denoted $B_{1}+B_{2}$ and their sequential composition by $B_{1} \gg B_{2}$. $B_{1} \|_{G} B_{2}$ denotes parallel composition where actions in $G \cup\{\delta\}$ $(G \subseteq$ Act $)$ are synchronization actions. $\| \mid$ abbreviates $\| \varnothing$, i.e., parallel composition without synchronization. $B[H]$ denotes the relabelling of $B$ according to $H$ where $H(\tau)=\tau, H(\delta)=\delta$ and for $a \in$ Act $: H(a) \neq \tau$ and $H(a) \neq \delta . B \backslash G$ denotes hiding. $B_{1}\left[>B_{2}\right.$ denotes the disruption of $B_{1}$ by $B_{2}$; i.e., $B_{1}$ may at any point of its execution disrupted by $B_{2}$, unless it terminated. Finally, $P$ denotes a process instantiation where a behaviour is considered in the context of a set of process definitions of the form $P:=B$ where $B$ possibly contains occurrences of $P$. The precedences of the composition operators are, in decreasing binding order: ; , , $\|$, $[>,>>, \backslash$ and []. Trailing $\mathbf{0 s}$ are usually omitted.

The standard (interleaving) semantics of PA is presented in Table 1 where $G^{\delta}$ denotes $G \cup\{\delta\}$.

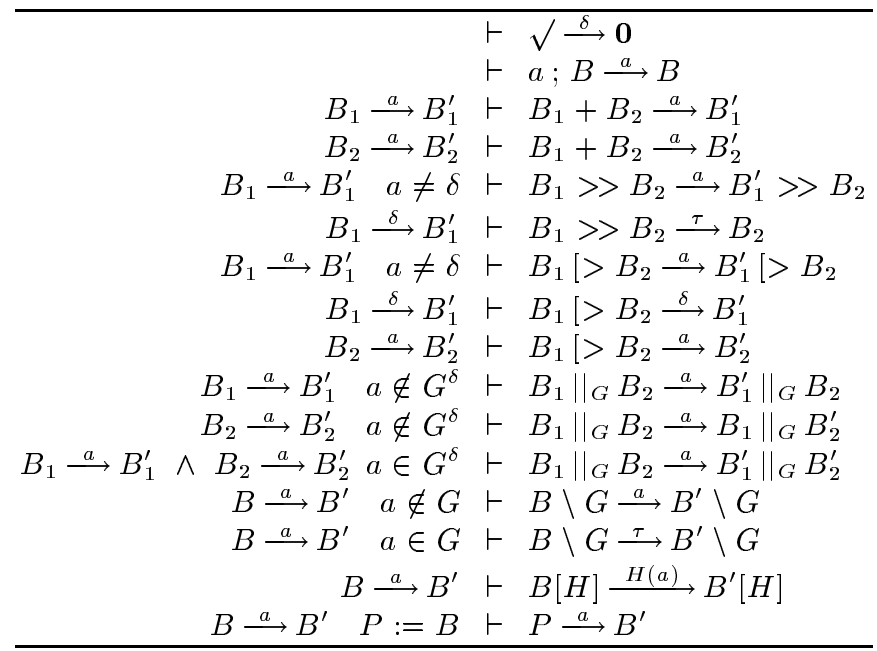

Table 1. Structured operational semantics of PA. 
The real-time variant of $\mathrm{PA}$, baptized $\mathrm{PA}_{R}$, is generated by the following grammar:

$$
\begin{aligned}
B::= & \mathbf{0}|\sqrt{ }|(T) a ; B|B+B| B \|_{G} B|B[H]| B \backslash G|B>>B| \\
& B[>B|B \triangleright B| B \stackrel{b}{\triangleright} B \mid P .
\end{aligned}
$$

We use Time $=\mathbb{R}^{+} \cup\{0, \infty\}$ as time domain, $T$ to range over $\mathcal{P}$ (Time), and $t$ to range over Time. $(T) a ; B$ denotes the timed action-prefix of $a$ and $B$ where $a$ is allowed (but not forced) to occur at some $t \in T$. We write $(t) a$ for $([t, \infty)) a$ and $a$ for (0) $a . B_{1} \stackrel{t}{\triangleright} B_{2}$ denotes the timeout of $B_{1}$ by $B_{2}$ at time $t$; initially it behaves like $B_{1}$, but if $B_{1}$ does not perform any action before $t$ (since the enabling of this behaviour) then the control is passed to $B_{2}$. At time $t$ a nondeterministic choice between $B_{1}$ and $B_{2}$ appears. $\triangleright$ is called a 'weak timeout' [21]. $>$ is a watchdog operator; initially $B_{1} \stackrel{t}{\downarrow} B_{2}$ behaves like $B_{1}$ but at time $t$ control is passed to $B_{2}$ provided $B_{1}$ is not yet successfully terminated. Note that in $B_{1} \stackrel{t}{\triangleright} B_{2}$ control is passed to $B_{2}$ only if $B_{1}$ does not perform any action-either internal or not-before $t$, whereas in $B_{1} \stackrel{t}{t} B_{2}$ control is passed to $B_{2}$ at time $t$, regardless of the activities of $B_{1}$ until time $t$ (with the exception of termination).

The synchronization principle is that an action can only occur when all participants are ready to engage in it. Thus, for instance, in $a ;\left(T_{1}\right) b \|_{\{a, b\}} a ;\left(T_{2}\right) b$, action $b$ is enabled at any time in $t_{a}+T_{1} \cap t_{a}+T_{2}=t_{a}+\left(T_{1} \cap T_{2}\right)$, where $t+T$ denotes $\left\{t+t^{\prime} \mid\right.$ $\left.t^{\prime} \in T\right\}$. Notice that synchronizations may become impossible due to incompatible timing constraints in the participating behaviours. For instance, if $T_{1} \cap T_{2}=\varnothing$, action $b$ can never occur.

\section{$3 \quad$ Extended bundle event structures}

Extended bundle event structures (or, simply: event structures) consist of events labelled with actions (an event modelling the occurrence of its action), together with relations of causality and conflict between events. System runs can be modelled as partial orders of events satisfying certain constraints posed by the causality and conflict relations between the events.

Conflict is an asymmetric ${ }^{4}$ binary relation, denoted $\rightsquigarrow$, between events and the intended meaning of $e \rightsquigarrow e^{\prime}$ is that (i) if $e^{\prime}$ occurs it disables the occurrence of $e$, and (ii) if $e$ and $e^{\prime}$ both occur in a single system run then $e$ causally precedes $e^{\prime}$. Causality is represented by a relation between a set $X$ of events, that are pairwise in conflict, and an event $e$. The interpretation is that if $e$ happens in a system run, exactly one event in $X$ has happened before (and caused $e$ ). This enables us to uniquely define a causal ordering between the events in a system run. When there is neither a conflict nor a causal relation between events they are independent. Once enabled, independent events can occur in any order or in parallel.

\footnotetext{
${ }^{4}$ The term asymmetric does not mean that $e \rightsquigarrow e^{\prime} \Rightarrow e^{\prime} \not \ngtr e$ as it might suggest. $e \rightsquigarrow e^{\prime}$ and $e^{\prime} \rightsquigarrow e$ is allowed and is equivalent with $e \# e^{\prime}$, the usual symmetric conflict in event structures. The terminology 'asymmetric' is adopted from Langerak [17] and Pinna \& Poigné [22].
} 
Definition 1. An (extended bundle) event structure $\mathcal{E}$ is a quadruple $(E, \rightsquigarrow, \mapsto, l)$ with $E$, a set of events, $\rightsquigarrow \subseteq E \times E$, the (irreflexive) asymmetric conflict relation, $\mapsto \subseteq \mathcal{P}(E) \times E$, the bundle relation, and $l: E \longrightarrow L$, the action-labelling function, where $L$ is a set of action labels, such that

$$
\forall X \subseteq E, e \in E: X \mapsto e \Rightarrow\left(\forall e^{\prime}, e^{\prime \prime} \in X: e^{\prime} \neq e^{\prime \prime} \Rightarrow e^{\prime} \rightsquigarrow e^{\prime \prime}\right)
$$

The constraint specifies that for bundle $X \mapsto e$ all events in $X$ are in mutual conflict. Event structures are graphically represented in the following way. Events are denoted as dots; near the dot the action label is given. $e \rightsquigarrow e^{\prime}$ is indicated by a dotted arrow from $e$ to $e^{\prime}$. A bundle $(X, e)$ is indicated by drawing an arrow from each event in $X$ to $e$ and connecting all arrows by small lines. We denote an event labelled $a$ by $e_{a}$. EBES denotes the class of event structures; $\mathcal{E}$ ranges over EBES.

In the sequel we adopt the following notations. For sequences $\sigma=x_{1} \ldots x_{n}$, let $\bar{\sigma}$ denote the set of elements in $\sigma$, that is, $\bar{\sigma} \triangleq\left\{x_{1}, \ldots, x_{n}\right\}$, and let $\sigma_{i}$ denote the prefix of $\sigma$ up to the $(i-1)$-th element, that is, $\sigma_{i} \triangleq x_{1} \ldots x_{i-1}$, for $0<i \leqslant n+1$. For $\sigma$ a sequence of events $e_{1} \ldots e_{n}$ we define $\operatorname{cfl}(\sigma) \triangleq\left\{e \in E \mid \exists e_{i} \in \bar{\sigma}: e \rightsquigarrow e_{i}\right\}$ and $\operatorname{sat}(\sigma) \triangleq\{e \in E \mid \forall X \subseteq E: X \mapsto e \Rightarrow X \cap \bar{\sigma} \neq \varnothing\} \cdot \operatorname{cfl}(\sigma)$ is the set of events that are disabled by some event in $\sigma$. sat $(\sigma)$ is the set of events that have a causal predecessor in $\sigma$ for all bundles pointing to them. That is, for events in $\operatorname{sat}(\sigma)$ all bundles are 'satisfied'. Let en $(\sigma) \triangleq \operatorname{sat}(\sigma) \backslash(\operatorname{cfl}(\sigma) \cup \bar{\sigma})$.

Event traces consist of distinct events (i.e., $\left.e_{i} \notin \overline{\sigma_{i}}\right)$ and are conflict-free $\left(e_{i} \notin \mathrm{cfl}\left(\sigma_{i}\right)\right)$, for obvious reasons. In addition, each event in the event trace is preceded in the sequence by a causal predecessor for each bundle pointing to it (i.e., $e_{i} \in \operatorname{sat}\left(\sigma_{i}\right)$ ).

Definition 2. An event trace $\sigma$ of $\mathcal{E}$ is a sequence of events $e_{1} \ldots e_{n}$ with $e_{i} \in \operatorname{en}\left(\sigma_{i}\right)$, for all $0<i \leqslant n$. Let $T(\mathcal{E})$ denote the set of event traces of $\mathcal{E}$.

Example 1. Figure 1(a) has bundles $\left\{e_{a}\right\} \mapsto e_{c},\left\{e_{b}\right\} \mapsto e_{c},\left\{e_{b}\right\} \mapsto e_{d}$, and a symmetric conflict between $e_{c}$ and $e_{d}$. In Figure $1(\mathrm{~b})$ we have $\left\{e_{a}, e_{a}^{\prime}\right\} \mapsto e_{b},\left\{e_{a}\right\} \mapsto$ $e_{x}$ and $\left\{e_{a}^{\prime}\right\} \mapsto e_{y}$. Some event traces of Figure 1(a) are $e_{a} e_{b} e_{c}, e_{b} e_{d} e_{a}$ and $e_{b} e_{a}$.

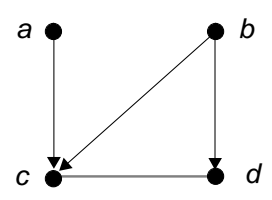

(a)

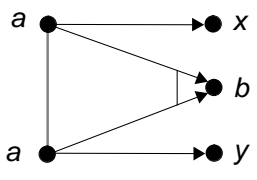

(b)

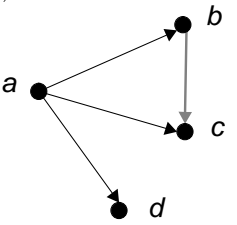

(c)

Fig. 1. Some example event structures.

Event structures can be used to provide a noninterleaving semantics to PA in a compositional way. For finite behaviours this is defined in Appendix A. The expressions corresponding to Figure 1 are as follows: (a) $a ; c \|_{\{c\}} b ;(c+d),(\mathrm{b})(a ; x\|\| a ; y)$ $\|_{\{a\}}(a ; b)$, and $(c) a ;((b[>c)\|\| d)$. 


\section{Real-time event structures}

Time is added to bundle event structures in two ways. To specify the relative delay between causally dependent events time is associated to bundles, and in order to facilitate the specification of timing constraints on events that have no bundle pointing to them (i.e., the initial events), time is also associated to events. Though it seems sufficient to only have time labels for initial events, synchronization of events makes it necessary to allow for equipping all events with time labels, including the non-initial ones. ${ }^{5}$

We assume mappings $\mathcal{T}$ and $\mathcal{D}$ to associate a set of time instants, to bundles and events, respectively. A bundle $(X, e)$ with $\mathcal{T}((X, e))=T$ is denoted by $X \stackrel{T}{\mapsto} e$; its interpretation is that if an event in $X$ has happened at a certain time, then $e$ is enabled $t$ time units later, for any $t \in T$. The interpretation of event $e$ with $\mathcal{D}(e)=T$ is that $e$ can happen at any $t \in T$ from the beginning of the system, usually assumed to be time 0 .

In order to specify timeout mechanisms we use urgent events. Urgent events are different from other events in the sense that they are forced to occur once they are enabled.

Definition 3. A real-time event structure $\Gamma$ is a quadruple $\langle\mathcal{E}, \mathcal{D}, \mathcal{T}, \mathcal{U}\rangle$ with $\mathcal{E}$, an event structure $(E, \rightsquigarrow, \mapsto, l), \mathcal{D}: E \longrightarrow \mathcal{P}$ (Time), the event delay function, $\mathcal{T}: \mapsto$ $\longrightarrow \mathcal{P}$ (Time), the bundle delay function, and $\mathcal{U}: E \longrightarrow$ Bool, the urgency predicate such that for all $e \in E$ with $\mathcal{U}(e)$ :

1. $\forall e^{\prime} \in E, X \subseteq E:\left(\left(e^{\prime} \rightsquigarrow e \vee e \rightsquigarrow e^{\prime}\right) \wedge X \mapsto e\right) \Rightarrow\left(X \mapsto e^{\prime} \vee X \rightsquigarrow e^{\prime}\right)$

2. $\exists t \in$ Time : $\mathcal{D}(e) \subseteq[t, t] \vee(\exists X \subseteq E: X \stackrel{T}{\mapsto} e \wedge T \subseteq[t, t])$.

Here, $X \rightsquigarrow e^{\prime}$ equals $\left(\forall e^{\prime \prime} \in X: e^{\prime \prime} \rightsquigarrow e^{\prime}\right)$. Note that $\varnothing \rightsquigarrow e^{\prime}$ for all $e^{\prime}$.

The first constraint requires that the enablings of an urgent event $e$ are either contained in the enablings of an event $e^{\prime}$ that it disables, i.e., $e^{\prime} \rightsquigarrow e$, or that an enabling of $e$ is disabled by $e^{\prime}$ (the case $e \rightsquigarrow e^{\prime}$ is identical). This constraint enforces that as soon as $e^{\prime}$ is enabled either $e$ is also enabled (provided $e$ is not disabled in another way), or is permanently disabled, since some enabling of $e$ is disabled (by $e^{\prime}$ ). As a result the global impact of urgent events is limited; see also [16]. Thus, in order to decide whether $e^{\prime}$ can occur-once it is enabled-it suffices to consider the local (and urgent) disablings of $e^{\prime}$.

The second constraint ensures that urgent events are enabled at a single time instant, if ever. The motivation for this constraint is that urgent events are used for the sole

\footnotetext{
${ }^{5}$ Alternatively, we could explicitly model the start of the system by some fictitious event, $\omega$ say. Then the time associated to event $e$ can be considered as the time associated to the bundle pointing from the fictitious event to $e$. We do not consider the introduction of such event $\omega$ since the definitions become more complex- $\omega$ has to be treated differently than 'normal' events - and proof obligations become more severe-e.g. one has to prove that bundles $X \mapsto e$ satisfy $X=\{\omega\}$, or $\omega \notin X$ and $e \neq \omega$.
} 
purpose of modelling timeouts, and a timeout typically can appear at a single time instant only.

Let $\mathrm{EBES}_{R}$ denote the class of real-time event structures. Bundle and event delays are depicted near to a bundle and event, respectively. Urgent events are denoted by open dots, other events by closed dots. Zero delays are omitted.

For events that have more than one bundle pointing to them we take the following interpretation. Consider $\left\{e_{a}\right\} \stackrel{T}{\longmapsto} e_{c}$ and $\left\{e_{b}\right\} \stackrel{T^{\prime}}{\longmapsto} e_{c}$. Then, if $e_{a}$ happens at time $t_{a}$ and $e_{b}$ at time $t_{b}$, then $e_{c}$ is enabled at any $t \in\left(t_{a}+T\right) \cap\left(t_{b}+T^{\prime}\right)$. When the intersection of two (or more) sets of time instants is empty this means that the event at hand cannot occur at any time and will be permanently disabled.

The notion of timed event trace is defined as a generalization of the notion of event trace. A timed event $(e, t)$ denotes that $e$ happened at time $t$. For sequences of timed events $\sigma=\left(e_{1}, t_{1}\right) \ldots\left(e_{n}, t_{n}\right)$ let $[\sigma]$ denote the sequence of events in $\sigma$, i.e., $[\sigma] \triangleq e_{1} \ldots e_{n}$. Let time $(\sigma, e)$ denote the set of time instants at which $e \in \operatorname{en}([\sigma])$ could happen, given that each event $e_{i}$ in timed event trace $\sigma$ occurred at time $t_{i}$. Event $e$ can occur if (i) its absolute delay $\mathcal{D}(e)$ is respected, (ii) the time relative to all its immediate causal predecessors is respected, and (iii) for each event $e_{j}$ with $e_{j} \rightsquigarrow e$ we have that $e$ occurs at at least $t_{j}$. (ii) and (iii) take care of the fact that events cannot occur before their causes, i.e., they entail that causal ordering implies temporal ordering. So,

$$
\begin{aligned}
\operatorname{time}(\sigma, e) \triangleq & \bigcap\left(\{\mathcal{D}(e)\} \cup H_{1} \cup H_{2}\right) \text { where } \\
& H_{1}=\left\{t_{j}+T \mid \exists X \subseteq E: X \stackrel{T}{ } e^{\wedge} \wedge X \cap \overline{[\sigma]}=\left\{e_{j}\right\}\right\} \\
& H_{2}=\left\{\left[t_{j}, \infty\right) \mid \exists e_{j} \in \overline{[\sigma]}: e_{j} \rightsquigarrow e\right\} .
\end{aligned}
$$

The notion of timed event trace is now defined as follows. Let $\operatorname{Min}(T)$ denote the minimum of set $T$. For $T=\varnothing, \operatorname{Min}(T) \triangleq \infty$.

Definition 4. A timed event trace of $\Gamma=\langle\mathcal{E}, \mathcal{D}, \mathcal{T}, \mathcal{U}\rangle$ is a sequence $\sigma$ of timed events $\left(e_{1}, t_{1}\right) \ldots\left(e_{n}, t_{n}\right)$ with $e_{i} \in E, t_{i} \in$ Time, satisfying $e_{1} \ldots e_{n} \in T(\mathcal{E}), t_{i} \in$ time $\left(\sigma_{i}, e_{i}\right)$, for all $0<i \leqslant n$, and

$$
\forall i, e:\left(e \in \operatorname{en}\left(\left[\sigma_{i}\right]\right) \wedge \mathcal{U}(e) \wedge\left(e_{i} \rightsquigarrow e \vee e \rightsquigarrow e_{i}\right)\right) \Rightarrow t_{i} \leqslant \operatorname{Min}\left(\operatorname{time}\left(\sigma_{i}, e\right)\right) .
$$

Let $T_{T}(\Gamma)$ denote the set of timed event traces of $\Gamma$. The first two constraints are self-explanatory. The third constraint takes care of the fact that urgent events may prevent the events that they disable (or by which they are disabled) to occur after a certain time. That is, event $e_{i}$ can occur at time $t_{i}$ provided there is no enabled urgent event $e$ that disables $e_{i}$ (or that is disabled by $e_{i}$ ) and that (if it occurs) must occur before $t_{i}$.

Example 2. Figure 2 depicts a real-time event structure with $\mathcal{T}\left(\left(\left\{e_{a}\right\}, e_{c}\right)\right)=[3,7]$, $\mathcal{T}\left(\left(\left\{e_{b}\right\}, e_{c}\right)\right)=[5,12]$ and $\mathcal{T}\left(\left(\left\{e_{b}\right\}, e_{d}\right)\right)=\{2,4,6, \ldots\}$. Event delays are all zero. For the following sequences of timed events the conditions are given under which they are timed event traces of Figure 2(a):

$$
\begin{aligned}
& \left(e_{a}, t_{a}\right)\left(e_{b}, t_{b}\right)\left(e_{d}, t_{d}\right) \text { if } t_{d} \in\left\{t_{b}+2, t_{b}+4, \ldots\right\}, \text { and } \\
& \left(e_{a}, t_{a}\right)\left(e_{b}, t_{b}\right)\left(e_{c}, t_{c}\right) \text { if } \max \left(t_{a}+3, t_{b}+5\right) \leqslant t_{c} \leqslant \min \left(t_{a}+7, t_{b}+12\right) .
\end{aligned}
$$




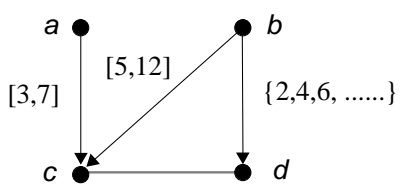

(a)

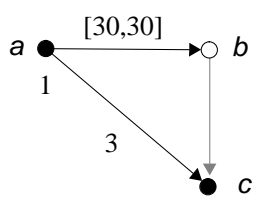

(b)

Fig. 2. Two real-time event structures.

For Figure 2(b) we obtain:

$$
\begin{aligned}
\left(e_{a}, t_{a}\right)\left(e_{c}, t_{c}\right) & \text { if } t_{a} \geqslant 1 \wedge t_{a}+3 \leqslant t_{c} \leqslant t_{a}+30, \text { and } \\
\left(e_{a}, t_{a}\right)\left(e_{b}, t_{b}\right)\left(e_{c}, t_{c}\right) & \text { if } t_{a} \geqslant 1 \wedge t_{b}=t_{a}+30 \wedge t_{c} \geqslant \max \left(t_{a}+3, t_{b}\right) .
\end{aligned}
$$

Timed event traces do respect causality, but not necessarily time. That is, two (or more) independent events can occur in a trace in either order regardless of their timing. For example, $\left(e_{b}, 1\right)\left(e_{a}, 3\right)$ and $\left(e_{a}, 3\right)\left(e_{b}, 1\right)$ are timed event traces of Figure 2. The possible choices correspond to the possible interleavings of the causally independent events. Since the causal ordering between events implies their temporal ordering the causal ordering can never contradict the temporal order.

The following result implies that for any ill-timed event trace $\sigma$ there exists a corresponding time-consistent event trace $\sigma^{\prime}$, that can be obtained from $\sigma$ by swapping repeatedly ill-timed pairs of timed events, yielding $\bar{\sigma}=\bar{\sigma}^{\prime}$.

Theorem 5. For $t^{\prime}<t: \sigma(e, t)\left(e^{\prime}, t^{\prime}\right) \sigma^{\prime} \in T_{T}(\Gamma) \Rightarrow \sigma\left(e^{\prime}, t^{\prime}\right)(e, t) \sigma^{\prime} \in T_{T}(\Gamma)$.

Note that the reverse implication does not hold; for instance, if $e$ causally depends on $e^{\prime}$ then the order of events $e^{\prime} e$ in a trace cannot be reversed since this would contradict their causal ordering. For a more extensive discussion on ill-timed traces we refer to $[1,2]$.

\section{Event structure semantics}

This section presents a causality-based semantics for $\mathrm{PA}_{R}$ using real-time event structures. We define a mapping $\mathcal{E}_{R} \llbracket \rrbracket: \mathrm{PA}_{R} \longrightarrow \mathrm{EBES}_{R}$. For convenience we use the denotational semantics $\mathcal{E}^{\prime} \llbracket \rrbracket$ for the untimed case which is defined in Appendix A.

Definition 6. $\Phi: \mathrm{PA}_{R} \longrightarrow \mathrm{PA}$ is defined as follows:

$$
\begin{aligned}
\Phi(\mathbf{0}) & \triangleq \mathbf{0} \\
\Phi(\sqrt{ }) & \triangleq \sqrt{ } \\
\Phi((T) a ; B) & \triangleq a ; \Phi(B) \\
\Phi\left(B_{1} \text { op } B_{2}\right) & \triangleq \Phi\left(B_{1}\right) \text { op } \Phi\left(B_{2}\right) \text { for op } \in\left\{+, \|_{G},>>,[>\}\right. \\
\Phi(\text { op } B) & \triangleq \text { op } \Phi(B) \text { for op } \in\{\backslash,[]\} \\
\Phi\left(B_{1} \triangleright^{t} B_{2}\right) & \triangleq \Phi\left(B_{1}\right)+\tau ; \Phi\left(B_{2}\right) \\
\Phi\left(B_{1} \stackrel{t}{ } B_{2}\right) & \triangleq \Phi\left(B_{1}\right)\left[>\Phi\left(B_{2}\right) .\right.
\end{aligned}
$$


$\Phi(B)$ is the untimed behaviour corresponding to $B$ obtained by omitting all time annotations in $B$ and converting $\triangleright$ and $\boldsymbol{\sim}$ into + and $[>$, respectively. The purpose of the internal event introduced by the timeout operator will be explained later on.

In the rest of this section let $\mathcal{E}_{R} \llbracket B_{i} \rrbracket=\Gamma_{i}=\left\langle\mathcal{E}_{i}, \mathcal{D}_{i}, \mathcal{T}_{i}, \mathcal{U}_{i}\right\rangle$, for $i=1,2$, with $\mathcal{E}_{i}=\left(E_{i}, \rightsquigarrow_{i}, \mapsto_{i}, l_{i}\right)$ and $E_{1} \cap E_{2}=\varnothing$. The functions init and exit which denote the set of initial and termination events, respectively, are defined for event structures in Appendix A and are used for real-time event structures in the same way. Let $E_{U}$ denote the (infinite) universe of events.

Definition 7. $\mathcal{E}_{R} \llbracket \rrbracket: \mathrm{PA}_{R} \longrightarrow \mathrm{EBES}_{R}$ is defined for $\mathbf{0}, \sqrt{ }$, and $(T) a$; as follows:

$$
\begin{aligned}
\mathcal{E}_{R} \llbracket \mathbf{0} \rrbracket & \triangleq\left\langle\mathcal{E}^{\prime} \llbracket \Phi(\mathbf{0}) \rrbracket, \varnothing, \varnothing, \varnothing\right\rangle \\
\mathcal{E}_{R} \llbracket \sqrt{ } \rrbracket & \triangleq\left\langle\mathcal{E}^{\prime} \llbracket \Phi(\sqrt{ }) \rrbracket,\left\{\left(e_{\delta}, \text { Time }\right)\right\}, \varnothing,\left\{\left(e_{\delta}, \text { false }\right)\right\}\right\rangle \\
\mathcal{E}_{R} \llbracket(T) a ; B_{1} \rrbracket & \triangleq\left\langle\left(E, \rightsquigarrow{ }_{1}, \mapsto, l_{1} \cup\left\{\left(e_{a}, a\right)\right\}\right), \mathcal{D}, \mathcal{T}, \mathcal{U}\right\rangle \text { where } \\
E & =E_{1} \cup\left\{e_{a}\right\} \text { for } e_{a} \in E_{U} \backslash E_{1} \\
\mapsto & =\mapsto_{1} \cup\left(\left\{\left\{e_{a}\right\}\right\} \times E_{1}\right) \\
\mathcal{D} & =\left\{\left(e_{a}, T\right)\right\} \cup\left(E_{1} \times\{\text { Time }\}\right) \\
\mathcal{T} & =\mathcal{T}_{1} \cup\left\{\left(\left(\left\{e_{a}\right\}, e\right), \mathcal{D}_{1}(e)\right) \mid e \in E_{1}\right\} \\
\mathcal{U} & =\mathcal{U}_{1} \cup\left\{\left(e_{a}, \text { false }\right)\right\} .
\end{aligned}
$$

The semantics of $\mathbf{0}$ and $\sqrt{ }$ is self-explanatory. In $\mathcal{E}_{R} \llbracket(T) a ; B_{1} \rrbracket$ a bundle is introduced from a new event $e_{a}$ (labelled $a$ ) to all events in $\Gamma_{1}$. The delay of these events $e$ becomes relative to $e_{a}$, so each bundle $\left\{e_{a}\right\} \mapsto e$ is associated with a time delay $\mathcal{D}_{1}(e)$, and $\mathcal{D}(e)$ becomes Time. $\mathcal{D}\left(e_{a}\right)$ becomes $T$. In the untimed case it suffices to only introduce bundles from $e$ to the initial events of $\Gamma_{1}$, cf. Appendix A. The bundles to all events of $\Gamma_{1}$ that are introduced in the timed case are used for the sole purpose of making delays relative to $e_{a}$. Figure 3 , e.g., shows (a) $\mathcal{E}_{R} \llbracket B \rrbracket$, and (b) $\mathcal{E}_{R} \llbracket([2,7)) a ; B \rrbracket$.

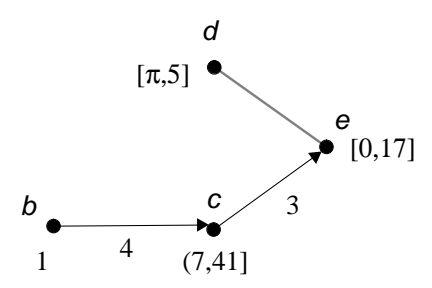

(a): $B$

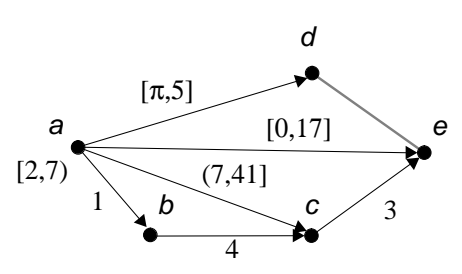

(b): $([2,7)) a ; B$

Fig. 3. Example of semantics for timed action prefix.

Definition 8. $\mathcal{E}_{R} \llbracket \rrbracket: \mathrm{PA}_{R} \longrightarrow \mathrm{EBES}_{R}$ is defined for $\backslash,[],+,>$ and $[>$ as follows: $\mathcal{E}_{R} \llbracket B_{1}$ op $B_{2} \rrbracket \triangleq\left\langle\mathcal{E}^{\prime} \llbracket \Phi\left(B_{1}\right.\right.$ op $\left.\left.B_{2}\right) \rrbracket, \mathcal{D}_{1} \cup \mathcal{D}_{2}, \mathcal{T}_{1} \cup \mathcal{T}_{2}, \mathcal{U}_{1} \cup \mathcal{U}_{2}\right\rangle$, op $\in\{+$, ; $\}$ $\mathcal{E}_{R} \llbracket$ op $B_{1} \rrbracket \triangleq\left\langle\mathcal{E}^{\prime} \llbracket \Phi\left(\right.\right.$ op $\left.\left.B_{1}\right) \rrbracket, \mathcal{D}_{1}, \mathcal{T}_{1}, \mathcal{U}_{1}\right\rangle$ for op $\in\{\backslash,[]\}$ 


$$
\begin{aligned}
\mathcal{E}_{R} \llbracket B_{1} \gg B_{2} \rrbracket & \triangleq\left\langle\left(E_{1} \cup E_{2}, \rightsquigarrow, \mapsto, l\right), \mathcal{D}, \mathcal{T}, \mathcal{U}_{1} \cup \mathcal{U}_{2}\right\rangle \text { where } \\
\rightsquigarrow & =\rightsquigarrow_{1} \cup \rightsquigarrow_{2} \cup\left\{\left(e, e^{\prime}\right) \mid e, e^{\prime} \in \operatorname{exit}\left(\Gamma_{1}\right) \wedge e \neq e^{\prime}\right\} \\
\mapsto & =\mapsto_{1} \cup \mapsto_{2} \cup\left(\left\{\operatorname{exit}\left(\Gamma_{1}\right)\right\} \times E_{2}\right) \\
l & =\left(\left(l_{1} \cup l_{2}\right) \backslash\left(\operatorname{exit}\left(\Gamma_{1}\right) \times\{\delta\}\right)\right) \cup\left(\operatorname{exit}\left(\Gamma_{1}\right) \times\{\tau\}\right) \\
\mathcal{D} & =\mathcal{D}_{1} \cup\left(E_{2} \times\{\operatorname{Time}\}\right) \\
\mathcal{T} & =\mathcal{T}_{1} \cup \mathcal{T}_{2} \cup\left\{\left(\left(\operatorname{exit}\left(\Gamma_{1}\right), e\right), \mathcal{D}_{2}(e)\right) \mid e \in E_{2}\right\} .
\end{aligned}
$$

For op equal to choice or disrupt $\mathcal{E}_{R} \llbracket B_{1}$ op $B_{2} \rrbracket$ is the untimed event structure of the corresponding expression in PA, $\mathcal{E}^{\prime} \llbracket \Phi\left(B_{1}\right.$ op $\left.B_{2}\right) \rrbracket$, where the timings of events and bundles in $\Gamma_{1}$ and $\Gamma_{2}$ are unaffected. Similarly, $\mathcal{E}_{R} \llbracket \rrbracket$ is defined for relabelling and hiding. The events of $\mathcal{E}_{R} \llbracket B_{1} \gg B_{2} \rrbracket$ are those in $E_{1} \cup E_{2}$. Bundles are introduced between the successful termination events of $\Gamma_{1}$ and the events in $\Gamma_{2}$. The reason for introducing bundles to all events of $\Gamma_{2}$ is to make the event delays in $\Gamma_{2}$ relative to the termination of $\Gamma_{1}$. This is similar as for timed action-prefix.

Now we consider parallel composition. Recall from Appendix A that events are pairs of events of $\Gamma_{1}$ and $\Gamma_{2}$, or with one component equal to $*$. The delay of an event is the maximum of the delays of its components that are different from $*$. The time associated with a bundle is equal to the maximum of the times associated with the bundles we get by projecting on the $i$-th components $(i=1,2)$ of the events in the bundle, if this projection yields a bundle in $\Gamma_{i}$.

For $E=\left(E_{1} \cup\{*\}\right) \times\left(E_{2} \cup\{*\}\right),\left(e_{1}, e_{2}\right) \in E$ and $X \subseteq E$ let for $i=1,2$ projection be defined as $\operatorname{pr}_{i}\left(\left(e_{1}, e_{2}\right)\right) \triangleq e_{i}$, if $e_{i} \neq *$ and $\operatorname{pr}_{i}(X) \triangleq\left\{\operatorname{pr}_{i}(e) \mid e \in X \cap \operatorname{dom}\left(\mathrm{pr}_{i}\right)\right\}$.

Definition 9. $\mathcal{E}_{R} \llbracket \rrbracket: \mathrm{PA}_{R} \longrightarrow \mathrm{EBES}_{R}$ is defined for $\|_{G}$ as follows:

$$
\begin{aligned}
\mathcal{E}_{R} \llbracket B_{1} \|_{G} B_{2} \rrbracket & \triangleq\left\langle\mathcal{E}^{\prime} \llbracket \Phi\left(B_{1} \|_{G} B_{2}\right) \rrbracket, \mathcal{D}, \mathcal{T}, \mathcal{U}\right\rangle \text { where } \\
\mathcal{D}\left(\left(e_{1}, e_{2}\right)\right) & =\mathcal{D}_{1}\left(e_{1}\right) \cap \mathcal{D}_{2}\left(e_{2}\right) \text { with } \mathcal{D}_{i}(*)=\text { Time. } \\
\mathcal{T}\left(\left(X,\left(e_{1}, e_{2}\right)\right)\right) & =\mathcal{T}_{1}\left(\left(p r_{1}(X), e_{1}\right)\right) \cap \mathcal{T}_{2}\left(\left(p r_{2}(X), e_{2}\right)\right) \text { with } \mathcal{T}_{i}\left(\left(\varnothing, e_{i}\right)\right)=\text { Time } \\
\mathcal{U}\left(\left(e_{1}, e_{2}\right)\right) & =\mathcal{U}_{1}\left(e_{1}\right) \vee \mathcal{U}_{2}\left(e_{2}\right) \text { with } \mathcal{U}_{i}(*)=\text { false. }
\end{aligned}
$$

Example 3. Consider the following timed behaviours

$$
\begin{aligned}
& B_{1}=([1,7)) a ;(5) b ; \mathbf{0} \|_{b}(\{1,3,6\}) c ;(7) b ; \mathbf{0} \\
& B_{2}=([4,9]) a ;(2) b ; \mathbf{0} \|_{b}(((4,27]) b ; \mathbf{0}+(3) d ; \mathbf{0}) .
\end{aligned}
$$

Figure 4 shows how $\mathcal{E}_{R} \llbracket B_{1} \|_{\{a, b\}} B_{2} \rrbracket$ is constructed from $\mathcal{E}_{R} \llbracket B_{1} \rrbracket$ and $\mathcal{E}_{R} \llbracket B_{2} \rrbracket$.
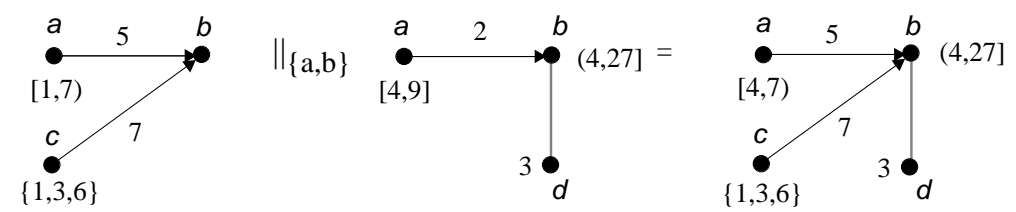

Fig. 4. Example of semantics for parallel composition. 
In $\mathcal{E}_{R} \llbracket B_{1} \stackrel{t}{\triangleright} B_{2} \rrbracket$ a new internal, urgent event $e_{\tau}$ is introduced that models the expiration of the timer. Since either the timer expires or $B_{1}$ performs an initial action before (or at) $t$, event $e_{\tau}$ is put in mutual conflict with all initial events of $\Gamma_{1}$. The events of $\Gamma_{2}$ can only occur after the timeout; this is modelled in the same way as for action-prefix: a bundle $\left\{e_{\tau}\right\} \mapsto e$ is introduced for all $e \in \Gamma_{2}$. The delay of these bundles is determined as in the action-prefix case. The event delay of $e_{\tau}$ becomes $[t, t]$ such that it can only occur at $t$ time units since the enabling of $\mathcal{E}_{R} \llbracket B_{1} \stackrel{t}{\triangleright} B_{2} \rrbracket$. So, $\mathcal{E}_{R} \llbracket B_{1} \stackrel{t}{\triangleright} B_{2} \rrbracket$ equals $\mathcal{E}_{R} \llbracket B_{1}+([t, t]) \tau ; B_{2} \rrbracket$ where $\tau$ is urgent.

Definition 10. $\mathcal{E}_{R} \llbracket \rrbracket: \mathrm{PA}_{R} \longrightarrow \mathrm{EBES}_{R}$ is defined for $\triangleright$ as follows:

$$
\begin{aligned}
\mathcal{E}_{R} \llbracket B_{1} \stackrel{t}{\triangleright} B_{2} \rrbracket & \triangleq\langle(E, \rightsquigarrow, \mapsto, l), \mathcal{D}, \mathcal{T}, \mathcal{U}\rangle \text { where } \\
E & =E_{1} \cup E_{2} \cup\left\{e_{\tau}\right\} \text { for some } e_{\tau} \in E_{U} \backslash\left(E_{1} \cup E_{2}\right) \\
\rightsquigarrow & =\rightsquigarrow_{1} \cup \rightsquigarrow_{2} \cup\left(\operatorname{init}\left(\Gamma_{1}\right) \times\left\{e_{\tau}\right\}\right) \cup\left(\left\{e_{\tau}\right\} \times \operatorname{init}\left(\Gamma_{1}\right)\right) \\
\mapsto & =\mapsto_{1} \cup \mapsto_{2} \cup\left(\left\{\left\{e_{\tau}\right\}\right\} \times E_{2}\right) \\
l & =l_{1} \cup l_{2} \cup\left\{\left(e_{\tau}, \tau\right)\right\} \\
\mathcal{D} & =\mathcal{D}_{1} \cup\left\{\left(e_{\tau},[t, t]\right)\right\} \cup\left(E_{2} \times\{\text { Time }\}\right) \\
\mathcal{T} & =\mathcal{T}_{1} \cup \mathcal{T}_{2} \cup\left\{\left(\left(\left\{e_{\tau}\right\}, e\right), \mathcal{D}_{2}(e)\right) \mid e \in E_{2}\right\} \\
\mathcal{U} & =\mathcal{U}_{1} \cup \mathcal{U}_{2} \cup\left\{\left(e_{\tau}, \text { true }\right)\right\} .
\end{aligned}
$$

Example 4. Let $B_{1}=(2) a ;(5) b\|\|([6,21)) c$ and $B_{2}=(3) d ;(2) g \|_{g}([27,41]) g$. Figure 5 illustrates how $\mathcal{E}_{R} \llbracket B_{1} \stackrel{12}{\triangleright} B_{2} \rrbracket$ is constructed from $\mathcal{E}_{R} \llbracket B_{1} \rrbracket$ and $\mathcal{E}_{R} \llbracket B_{2} \rrbracket$.

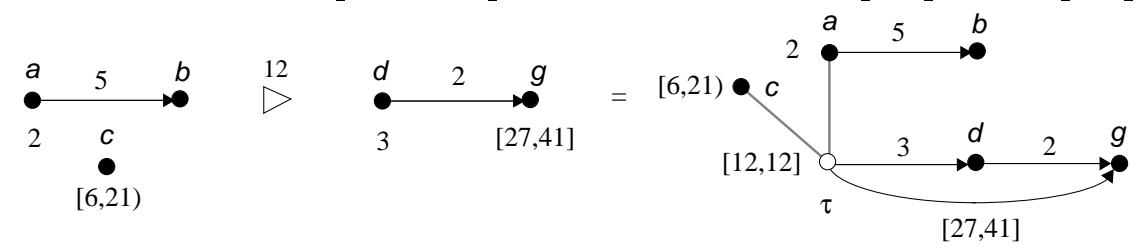

Fig. 5. Example of semantics for $\triangleright$.

A similar approach could be taken for the watchdog operator (using [ $>$ rather than + ), but there is also a possibility to model $B_{1} t B_{2}$ without using urgent events. Consider $\mathcal{E}_{R} \llbracket B_{1}\left[>B_{2} \rrbracket\right.$, i.e., the real-time event structure of $B_{1}\left[>B_{2}\right.$, and (i) restrict all event delays in $\Gamma_{1}$ by $[0, t]$ ensuring that these events can only occur at time $t$ at the latest, and (ii) postpone all events in $\Gamma_{2}$ by time $t$ such that these events can only occur from $t$ on.

Definition 11. $\mathcal{E}_{R} \llbracket \rrbracket: \mathrm{PA}_{R} \longrightarrow \mathrm{EBES}_{R}$ is defined for $>$ as follows:

$$
\begin{aligned}
\mathcal{E}_{R} \llbracket B_{1}{ }^{t} B_{2} \rrbracket & \left.\triangleq \mathcal{E}^{\prime} \llbracket \Phi\left(B_{1} \stackrel{t}{ }^{\prime} B_{2}\right) \rrbracket, \mathcal{D}, \mathcal{T}_{1} \cup \mathcal{T}_{2}, \mathcal{U}_{1} \cup \mathcal{U}_{2}\right\rangle \text { where } \\
\mathcal{D} & =\left\{\left(e, \mathcal{D}_{1}(e) \cap[0, t]\right) \mid e \in E_{1}\right\} \cup\left\{\left(e, t+\mathcal{D}_{2}(e)\right) \mid e \in E_{2}\right\} .
\end{aligned}
$$


Figure 6 shows how $\Gamma_{1}{ }^{12} \Gamma_{2}$ is constructed from $\Gamma_{1}$ and $\Gamma_{2}$.
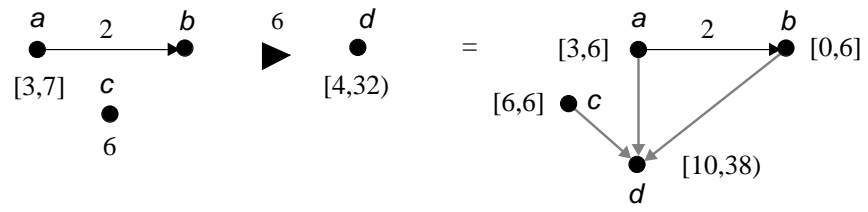

Fig. 6. Example of semantics for watchdog operator.

Theorem 12. $\forall B \in \mathrm{PA}_{R}: \mathcal{E}_{R} \llbracket B \rrbracket \in \mathrm{EBES}_{R}$.

\section{Recursion}

In this section we extend $\mathrm{PA}_{R}$ with recursion. To that end we extend the syntax with the construct $B::=P$ where $P$ denotes a process instantiation. We assume a behaviour is always considered in the context of a set of process definitions of the form $P:=B$ where $B$ is a behaviour possibly containing occurrences of $P$.

$\mathcal{E}_{R} \llbracket P \rrbracket$ for $P:=B$ is defined in the following way by using standard fixed point theory [27]. A complete partial order (c.p.o.) $\unlhd$ is defined on real-time event structures with the empty event structure (i.e., $\mathcal{E}_{R} \llbracket \mathbf{0} \rrbracket$ ) as the least element - . Then for each definition $P:=B$ a function $\mathcal{F}_{B}$ is defined that substitutes a real-time event structure for each occurrence of $P$ in $B$, interpreting all operators in $B$ as operators on real-time event structures. $\mathcal{F}_{B}$ is shown to be continuous, which means that $\mathcal{E}_{R} \llbracket P \rrbracket$ can be defined as the least upper bound (l.u.b.) of the chain (under $\unlhd$ ) -, $\mathcal{F}_{B}(-), \mathcal{F}_{B}\left(\mathcal{F}_{B}(-)\right), \ldots$ For this paper we just define the appropriate ordering $\unlhd$, the corresponding 1.u.b., and present the main results. Given these ingredients it is rather straightforward to define a continuous function $\mathcal{F}_{B}$. Further details can be found in [14].

Definition 13. Let $\Gamma_{i}=\left\langle\left(E_{i}, \rightsquigarrow_{i}, \mapsto_{i}, l_{i}\right), \mathcal{D}_{i}, \mathcal{T}_{i}, \mathcal{U}_{i}\right\rangle$ for $i=1,2$. Then $\Gamma_{1} \unlhd \Gamma_{2}$ iff $E_{1} \subseteq E_{2}, l_{1}=l_{2}\left\lceil E_{1}, \mathcal{D}_{1}=\mathcal{D}_{2}\left\lceil E_{1}, \mathcal{U}_{1}=\mathcal{U}_{2}\left\lceil E_{1}\right.\right.\right.$, and

1. $\rightsquigarrow_{1}=\rightsquigarrow_{2} \cap\left(E_{1} \times E_{1}\right)$

2. $\mapsto_{1}=\left\{\left(\left(X \cap E_{1}\right), e\right) \mid e \in E_{1} \wedge X \mapsto_{2} e\right\}$, and

3. $\forall e \in E_{1}: \mathcal{T}_{1}\left(\left(X \cap E_{1}, e\right)\right)=\mathcal{T}_{2}((X, e))$.

where $\uparrow$ denotes restriction. It is straightforward to verify that $\unlhd$ is a partial order with $-=\langle(\varnothing, \varnothing, \varnothing, \varnothing), \varnothing, \varnothing, \varnothing\rangle$ as least element. For conflicts we require that no new conflicts appear in $\Gamma_{2}$ between events that are already in $\Gamma_{1}$. Similarly, the second constraint forbids the introduction of bundles in $\Gamma_{2}$ pointing to events in $\Gamma_{1}$ for which there exists no projected bundle in $\Gamma_{1}$. Note that this constraint allows for bundles to grow in such a way that the old bundle is contained in the new one. The last constraint requires for those bundles to keep the same delay. 
The 1.u.b. of a chain $\Gamma_{1} \unlhd \Gamma_{2} \unlhd \ldots$, denoted $\bigsqcup_{i} \Gamma_{i}$, can be characterized as follows. For the set of events, conflicts, labeling function, and event delays we simply take the union of all events, conflicts, labellings and event delays of the event structures in the chain. As bundles may grow this approach does not apply to the set of bundles. Suppose some $\Gamma_{j}$ has bundle $X_{j} \mapsto_{j} e$. According to the definition of $\unlhd$ there is a series of bundles $X_{j} \mapsto_{j} e, X_{j+1} \mapsto_{j+1} e, \ldots$ satisfying $X_{k+1} \cap E_{k}=X_{k}$ for $k \geqslant j$. Then the 1.u.b. contains bundle $\left(\bigcup_{n} X_{j+n}\right) \mapsto e$. For $\Gamma_{1} \unlhd \Gamma_{2} \unlhd \ldots$ :

Definition 14. Let $\bigsqcup_{i} \Gamma_{i} \triangleq\left\langle\left(\bigcup_{i} E_{i}, \bigcup_{i} \rightsquigarrow_{i}, \mapsto, \bigcup_{i} l_{i}\right), \bigcup_{i} \mathcal{D}_{i}, \mathcal{T}, \bigcup_{i} \mathcal{U}_{i}\right\rangle$ with

$$
\begin{aligned}
& \mapsto=\left\{\left(\bigcup_{k} X_{k}, e\right) \mid \exists j:\left(\forall k \geqslant j: X_{k} \mapsto_{k} e \wedge X_{k+1} \cap E_{k}=X_{k}\right)\right\} \\
& \mathcal{T}=\left\{\left(\left(\bigcup_{k} X_{k}, e\right), T\right) \mid \exists j:\left(\forall k \geqslant j: X_{k} \stackrel{T}{\mapsto}_{k} e \wedge X_{k+1} \cap E_{k}=X_{k}\right)\right\} .
\end{aligned}
$$

Proposition 15. $\bigsqcup_{i} \Gamma_{i}$ is the least upper bound of chain $\Gamma_{1} \unlhd \Gamma_{2} \unlhd \ldots$

Proposition 16. For $\Gamma_{1} \unlhd \Gamma_{2} \unlhd \ldots$ a chain: $T_{T}\left(\bigsqcup_{i} \Gamma_{i}\right)=\bigcup_{i} \bigcap_{j \geqslant i} T_{T}\left(\Gamma_{j}\right)$.

Definition 17. For $P:=B$ a process definition let $\mathcal{E}_{R} \llbracket P \rrbracket \triangleq \bigsqcup_{i} \mathcal{F}_{B}^{i}(-)$.

Example 5. As an example of a recursive process definition in $\mathrm{PA}_{R}$ we consider

$$
P:=([3,5]) a ;((14) b ; P+(1) c ;([3, \pi)) d ; P)
$$

The first approximation of the real-time event structure semantics of this definition is - , the empty structure. The second approximation $\mathcal{F}_{B}(-)$ is depicted in Figure $7(\mathrm{a})$. By repeated substitution we obtain the real-time event structure depicted in Figure $7(b)$.

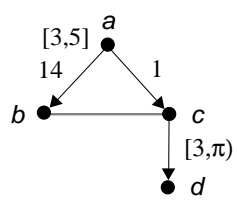

(a)

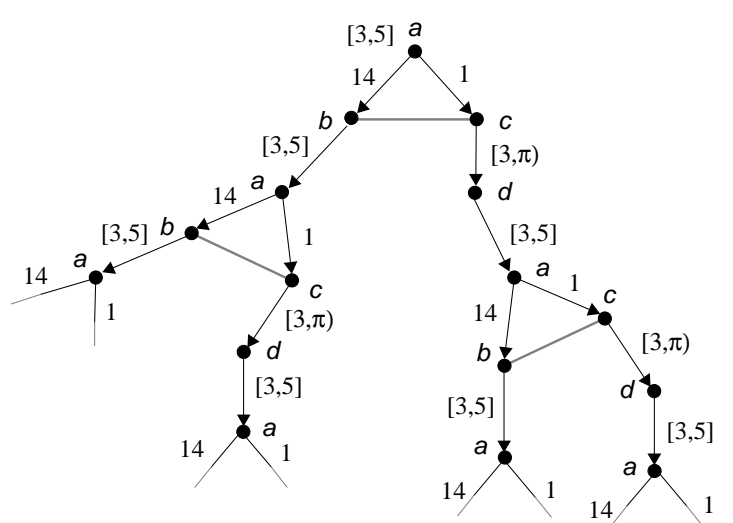

(b)

Fig. 7. Example of semantics for a recursive process definition in $\mathrm{PA}_{R}$. 


\section{Example: a time-constrained FIFO buffer}

We show how $\mathrm{PA}_{R}$ and real-time event structures can be used to specify real-time systems by treating a time-constrained first-in first-out (FIFO) buffer. This example is taken from [30]; the only difference is that we consider a buffer of infinite length. A simple way to specify a FIFO buffer is by using an abstract data type queue:

$$
\left.\operatorname{Fifo}(w: \text { queue }):=\sum_{x \in D}\left([w=\langle x\rangle\urcorner^{\prime}\right] \rightarrow r d_{x} ; \operatorname{Fifo}\left(w^{\prime}\right)+w r_{x} ; \operatorname{Fifo}(w \frown\langle x\rangle)\right) \quad .
$$

$D$ is a set of data values that can be buffered, $w r_{x}$ denotes the writing (i.e., insertion) of $x \in D$ into the buffer and $r d_{x}$ denotes the reading (i.e., removal) of $x$ from the buffer. $\sum$ is a generalized version of the choice operator; $\langle x\rangle$ denotes a singleton queue containing $x$ and $\frown$ denotes concatenation of queues. $[b] \rightarrow E$ denotes that $E$ can be executed if condition $b$ holds.

The FIFO buffer should model a communication network with the following timing constraints [30]: (i) message latency in the range of 2 to 5 time units; (ii) message input rate set to 1 message per time unit; (iii) message output rate of 1 message per two time units. These time constraints are maintained by the following processes:

$$
\begin{aligned}
& T D:=\left(w r_{x} ;([2,5]) r d_{x}\right) \| T D \\
& W r:=w r_{x} ; W r^{\prime} \text { where } W r^{\prime}:=(1) w r_{x} ; W r^{\prime} \\
& R d:=r d_{x} ; R d^{\prime} \text { where } R d^{\prime}:=(2) r d_{x} ; R d^{\prime} .
\end{aligned}
$$

The required buffer is obtained by putting these processes in parallel with Fifo:

$$
\text { Fifo }(\langle\rangle)\|R d\| W r \| T D
$$

where $\|$ is a shorthand for $\|_{\text {Act }}$, i.e., full synchronization. This specification strongly resembles the timed CSP specification in [30].

A problem with this specification is that it prescribes a mutual exclusion between reading and writing: at any moment one may either choose to read (provided the buffer is not empty) or to write. However, intuitively reading and writing should be to a certain extent independent. If the queue contains one or more elements, it should be possible to read them in parallel with writing new elements. The mutual exclusion constraint is especially unnatural if reading and writing take place at different locations (which is quite common in case of a communication network). We therefore propose a different way of modelling a time-constrained FIFO buffer in which we exploit the use of event structures as a partial order model:

$$
\begin{aligned}
\text { Cell } & :=w p ; \sum_{x \in D} w r_{x} ;\left((1) w n \| \mid([2,5]) r p ; r d_{x} ;(2) r n\right) \\
\text { Chain } & :=\left(\text { Cell } \|_{\{w n, r n\}} \text { Chain }[w p:=w n, r p:=r n]\right) \backslash\{w n, r n\} \\
\text { Buf } & :=\text { Chain } \backslash\{w p, r p\} .
\end{aligned}
$$

The real-time event structures corresponding to the Cell and Buf processes are depicted in Figure 8(a) and (b), respectively. The unlabelled, grey dots represent internal events. 


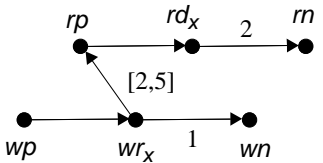

(a)

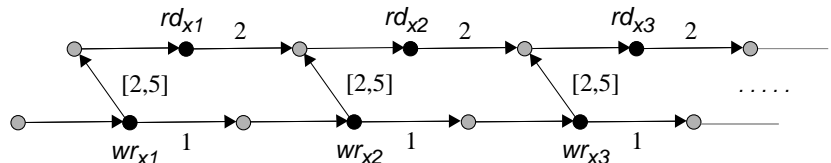

(b)

Fig. 8. Real-time event structure of a time-constrained FIFO buffer.

Process Cell describes a buffer cell allowing the writing and reading of a data value. The actions $w p$ and $r p$ ensure that the cell waits before writing resp. reading; $w n$ and $r n$ indicate the finish of writing and reading and are used in Chain to 'start' the next cell. Chain puts an unbounded number of cells in parallel using an appropriate renaming function. Finally, process Buf hides the write-previous and read-previous actions of the front cell.

Note that the $r d_{x}$ and $w r_{x}$ events in the latter specification are in principle timeunconstrained. It would be more natural to force these events to happen as soon as they are enabled. This can be done by using an explicit urgent operator; the treatment of such operator in our setting falls beyond the scope of this paper and can be found in [16].

\section{Event-based operational semantics}

Various timed process algebras are known based on an interleaving semantics. In order to compare our noninterleaving approach to these existing approaches and to investigate the 'compatibility' of our proposal with the standard (interleaving) semantics of LOTOS we present an event-based operational semantics for $\mathrm{PA}_{R}$. The basic idea is to define a transition system (in the sense of [23]) in which we keep track of the (times of) occurrence of actions rather than the actions themselves as is usual in structured operational semantics. This results in a timed event transition system. The approach is adopted from $[6,17]$ and is also applied in [16].

Each occurrence of an action-prefix, $\sqrt{ }$ and $\triangleright$ is subscripted with an arbitrary but unique event occurrence identifier, denoted by a Greek letter. These occurrence identifiers play the rôle of event names. For parallel composition new event names can be created. If $e$ is an event name of $B$ and $e^{\prime}$ an event name in $B^{\prime}$, then possible new names for events in $B \|_{G} B^{\prime}$ are $(e, *)$ and $\left(*, e^{\prime}\right)$ for unsynchronized events and $\left(e, e^{\prime}\right)$ for synchronized events.

The operational semantics defines a set of transition relations $\stackrel{(e, a, t)}{\longrightarrow} . B \stackrel{(e, a, t)}{\longrightarrow}$ $B^{\prime}$ denotes that behaviour $B$ can perform event $e$, labelled with action $a \in \operatorname{Act}^{\tau, \delta}$, at time $t \in$ Time, and subsequently evolve into $B^{\prime}$. The transition relation $\longrightarrow$ is the smallest relation closed under all inference rules listed in Table 2.

As a subsidiary notion let ut $(B)$ denote the set of time instants at which $B$ can initially perform an urgent event. Let $\mathrm{PA}_{R}^{+}$denote $\mathrm{PA}_{R}$ including the auxiliary operators ${ }^{t}[]$ and ${ }^{t}\{\}$ (see below). 
Definition 18. ut : $\mathrm{PA}_{R}^{+} \longrightarrow \mathcal{P}($ Time $)$ is defined by:

$$
\begin{aligned}
\operatorname{ut}\left({ }^{t}[B]\right) & \triangleq\left\{t^{\prime}+t \mid t^{\prime} \in \operatorname{ut}(B)\right\} \\
\operatorname{ut}\left(B_{1} \text { op } B_{2}\right) & \triangleq u t\left(B_{1}\right) \cup \operatorname{ut}\left(B_{2}\right) \text { for op } \in\left\{+,\left[>, \|_{G}\right\}\right. \\
\operatorname{ut}\left({ }^{t}\{B\}\right) & \triangleq\left\{t^{\prime} \in \operatorname{ut}(B) \mid t^{\prime} \geqslant t\right\} \\
\operatorname{ut}\left(B_{1} \gg B_{2}\right) & \triangleq \operatorname{ut}\left(B_{1}\right) \\
\operatorname{ut}(\text { op } B) & \triangleq \operatorname{ut}(B) \text { for op } \in\{\backslash,[]\} \\
\operatorname{ut}\left(B_{1} \triangleright^{t} B_{2}\right) & \triangleq \operatorname{ut}\left(B_{1}\right) \cup\{t\} \\
\operatorname{ut}\left(B_{1}{ }^{t} B_{2}\right) & \triangleq \operatorname{ut}\left(B_{1}\right) \cup \operatorname{ut}\left({ }^{t}\left[B_{2}\right]\right) \\
\operatorname{ut}(P) & \triangleq \operatorname{ut}(B) \text { for } P:=B .
\end{aligned}
$$

For all other syntactical constructs let ut $(B) \triangleq \varnothing$.

Let $\mathrm{mt}(B)$ abbreviate $\operatorname{Min}(\mathrm{ut}(B))$, where Min of the empty set equals $\infty$. In order to let ut be well defined we require process instantiations to occur in a weakly guarded way (i.e., they should become guarded after a finite number of substitutions of bodies for their process names).

As $\mathbf{0}$ cannot perform any transition there is no rule for this construct. $\sqrt{ }$ can perform the successful termination action $\delta$ at any time $t .(T) a_{\xi} ; B$ can perform event $\xi$ at time $t, t \in T$, and evolves into ${ }^{t}[B] \cdot{ }^{t^{\prime}}[B]$ can be considered as behaviour $B$ shifted $t^{\prime}$ time units in advance. That is, if $B$ can perform event $\xi$, say, at time $t$, then ${ }^{\prime}[B]$ can perform $\xi$ at time $t+t^{\prime}$. Note that ${ }^{\prime}[B]$ is only an auxiliary construct; it has no counterpart at the language level.

The rules for parallel composition in which no synchronization takes place, for hiding, and for relabelling are straightforward extensions of the untimed rules. Synchronization can only take place when both participants can perform an equally labelled event whose label is in the synchronization set $G$ (or equals $\delta$ ) at time $t$.

The rules for $\gg$ are also a straightforward extension of the rules for the untimed case except that in case $B_{1}$ performs a successful termination action $\delta$ at time $t$, then $B_{1} \gg B_{2}$ evolves into ${ }^{t}\left[B_{2}\right]$ rather than $B_{2}$. This represents that $t$ time units have been passed before $B_{2}$ can start with its execution. This is similar to the timed action-prefix case.

The rules for $B_{1}+B_{2}$ are somewhat adapted since (initial) urgent events in $B_{1}$ or $B_{2}$ can decide the choice. E.g., in

$$
\text { (12) } a+\left((18) b b^{5} \triangleright_{\chi}([1,7) c)\right.
$$

the event $\chi$ will occur at time 5 , and resolve the choice in favour of $B_{2}$. In general, if $B_{1}$ performs an event at time $t$ then $B_{1}+B_{2}$ can perform the same provided that $B_{2}$ cannot perform an urgent event at any time earlier, i.e., if $t \leqslant \operatorname{mt}\left(B_{2}\right)$. By symmetry, a similar condition is obtained for $B_{2}$ performing an event. Similar conditions appear for $[>, \triangleright$, and

For $B_{1}\left[>B_{2}\right.$ the rules are justified as follows. If $B_{1}$ performs an event at time $t$ and evolves into $B_{1}^{\prime}$ then $B_{1}\left[>B_{2}\right.$ can do the same while evolving into $B_{1}^{\prime}\left[{ }^{t}\left\{B_{2}\right\}\right.$. 


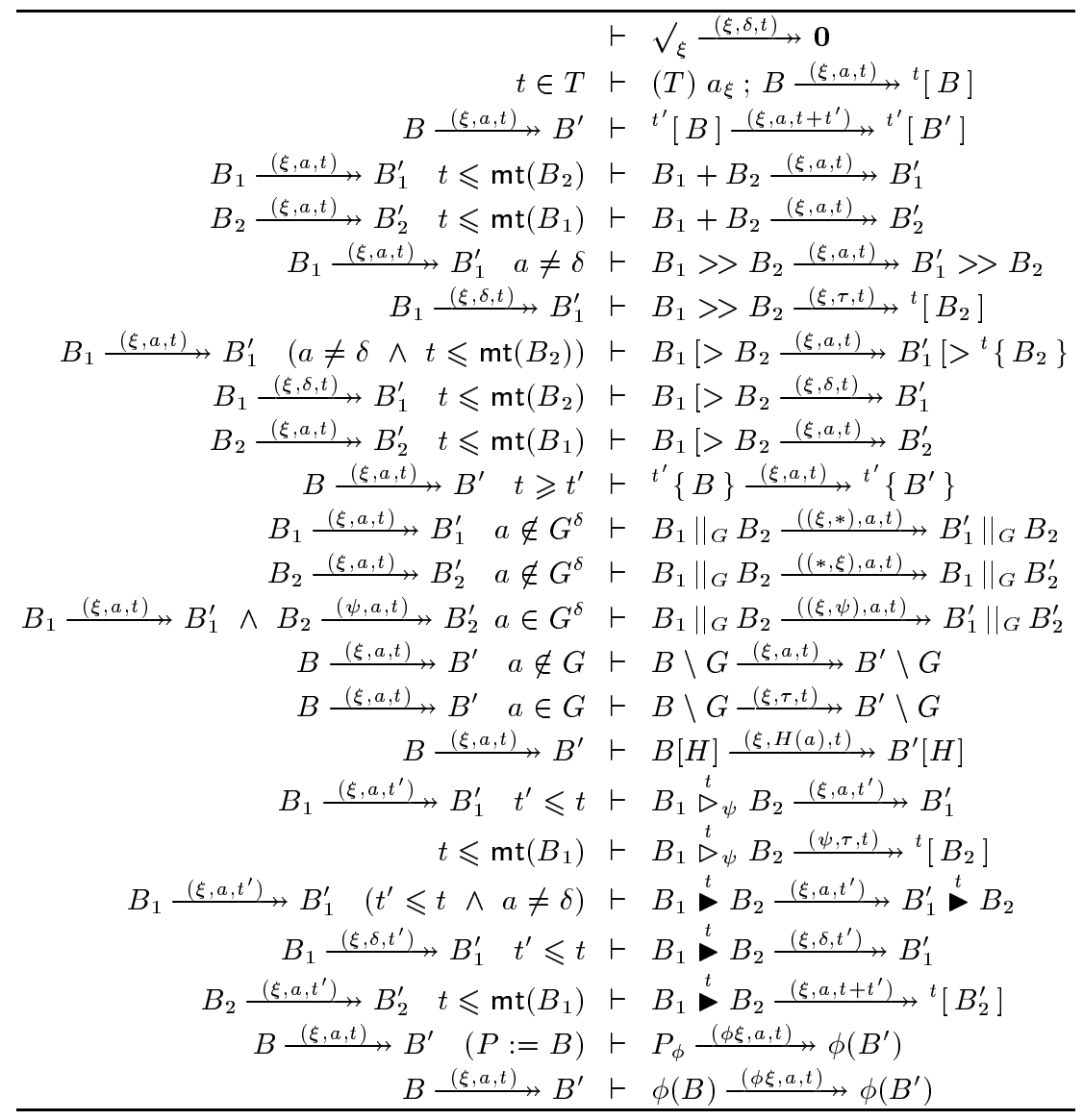

Table 2. Event-based operational semantics for $\mathrm{PA}_{R}$.

${ }^{t}\left\{B_{2}\right\}$ behaves like $B_{2}$ except that it is unable to perform events before $t$. This ensures that $B_{2}$ cannot disrupt $B_{1}^{\prime}\left[>B_{2}\right.$ by performing an event at time $t^{\prime}$, say, while $B_{1}$ has performed an event at time $t>t^{\prime}$. The other inference rules for disrupt are straightforward extensions of the rules for the untimed case (using similar conditions as for + ).

The inference rule for $t^{\prime}\{B\}$ is that if $B$ can perform an event at time $t$, then $t^{\prime}\{B\}$ can do so if $t \geqslant t^{\prime}$. Note that ${ }^{t^{\prime}}\{B\}$ is-like ${ }^{t^{\prime}}[B]$-an auxiliary operator that cannot be specified by the user.

If $B_{1}$ performs an event at time $t^{\prime}$, with $t^{\prime} \leqslant t$, and evolves into $B_{1}^{\prime}$ then $B_{1} \stackrel{t}{\triangleright_{\psi}} B_{2}$ can do the same; in this case the possibility that $B_{2}$ happens is dropped since $B_{1}$ has performed an action before (or at) time $t$. At time $t$ the timeout event $\psi$ can happen and the resulting behaviour is ${ }^{t}\left[B_{2}\right], B_{2}$ shifted $t$ time units in advance. This can only be done if $t \leqslant \mathrm{mt}\left(B_{1}\right)$. This condition ensures that $\psi$ is not performed if $B_{1}$ can perform an urgent event before $t$. E.g., in $\left(a \stackrel{7}{\triangleright_{\xi}} b\right) \stackrel{21}{\triangleright_{\psi}} c$ it prevents $\psi$ from 
happening (at time 21), since $\xi$ should have happened (at time 7 ).

If $B_{1}$ performs an event (which is not a successful termination event) at time $t^{\prime}$, with $t^{\prime} \leqslant t$, and evolves into $B_{1}^{\prime}$ then $B_{1} \stackrel{t}{\bullet} B_{2}$ can do the same while evolving into $B_{1}^{\prime} B_{2}$; the possibility for disruption (at time $t$ ) by $B_{2}$ remains. If $B_{1}$ terminates successfully at time $t^{\prime}, t^{\prime} \leqslant t$, disruption by $B_{2}$ becomes impossible (like for $B_{1}[>$ $B_{2}$ ). If $B_{2}$ performs an event at time $t^{\prime}$ and evolves into $B_{2}^{\prime}$ then $B_{1}{ }^{t} B_{2}$ can perform the same (provided $B_{1}$ cannot perform an urgent event before $t$ ) and evolves into ${ }^{t}\left[B_{2}^{\prime}\right], B_{2}^{\prime}$ shifted $t$ time units in time.

It is assumed that each process instantiation of $P$ is uniquely identified like all occurrences of action-prefix and $\sqrt{ }$. Different occurrences of the same process instantiation should produce different event transitions. In addition, event transitions cannot be repeated. For $P:=([2,7]) a_{\xi} ; P_{\phi}$ we first have an event transition with $(\xi, a, t)$ for $t \in[2,7]$; the next time that action $a$ occurs it should be labelled with a label different from $\xi$. These complications are resolved by using an event renaming operator that prefixes all events in a behaviour with a certain occurrence identifier. $\phi(B)$ is behaviour $B$ where all event identifiers in $B$ are prefixed with $\phi$.

Let $\operatorname{UE}(B)$ denotes the set of urgent events in $B$. (This function can easily be defined by induction on the structure of $B$ and is omitted here.)

Proposition 19. $\forall B \in \mathrm{PA}_{R}^{+}:(t \leqslant \mathrm{mt}(B)) \Leftrightarrow\left(\forall e \in \mathrm{UE}(B), t^{\prime}<t: B \stackrel{\left(e, \tau, t^{\prime}\right)}{\leftrightarrow} \leftrightarrow\right)$.

The consistency between the denotational and operational semantics of $\mathrm{PA}_{R}$ is given by

Theorem 20. $\forall B \in \mathrm{PA}_{R}: T_{T}\left(\mathcal{E}_{R} \llbracket B \rrbracket\right)=\left\{\sigma \mid \exists B^{\prime}: B \stackrel{\sigma}{\longrightarrow} B^{\prime}\right\}$.

\section{Conclusions and related work}

This paper concerns a real-time extension of (a variant of) event structures, a partialorder model for concurrent systems. The original incentives of our work are to study the expressiveness of event structures to effectively support the specification of distributed systems and to facilitate formal representation of performance and reliability aspects. A secondary aim is to (formally) relate the real-time extension of event structures to interleaving models for concurrency such that partial-order and interleaving models can be used coherently in the system design process and can be compared in a perspicuous way.

To achieve this we proposed a real-time variant of extended bundle event structures, used this model for providing a (noninterleaving) denotational semantics to a realtime process algebraic formalism that includes a timeout and watchdog operator, and constructed a corresponding event-based operational semantics. This shows that event structures are suitable for modelling real-time systems. Both semantics are characterized by the absence of any mechanism that explicitly forces the passage 
of time; time is treated as a parameter. The event-based operational semantics is a conservative extension of the standard interleaving operational semantics of LOTOS.

An interaction can take place if all participants can engage in it at the same time instant. The interaction cannot appear if such common time instant does not exist. Since in our model we do not have an explicit notion of the passage of time such an impossible interaction does not result in behaviours which do block the passage of time (so-called timelocks) in the entire system - even in causally independent parts! - but simply in the local impossibility to execute the event at hand.

The model based on timed-actions allows for the generation of ill-timed traces like in $[1,2]$. Recently, [11] proposed a timed process algebra with the theoretical CSP parallel operator that also includes ill-timed traces. In the proposals $[1,2,11]$ subprocesses have their independent local clock, and since local clocks are only synchronized at interaction, ill-timedness appears. We believe that the operational semantics presented in this paper is simpler by avoiding local clocks.

Ill-timedness is a phenomenon that is sometimes explicitly avoided by others (like in real-time ACP [4] and TIC [25]), since the precedence of timed events in the trace does not reflect the order in time. To our opinion ill-timed traces are not that obscure, since for each ill-timed trace there exists a corresponding time-consistent trace with the same timed events. Moreover, we think that the avoidance of them leads to a more complicated operational semantics.

Acknowledgements. The authors would like to thank Pedro d'Argenio and Arend Rensink for their suggestions.

\section{A Denotational semantics of PA}

In this appendix we provide the full definition of the causality-based semantics of PA. The initial events and successful termination events of an event structure are defined as follows: $\operatorname{init}(\mathcal{E}) \triangleq\{e \in E \mid \neg(\exists X \subseteq E: X \mapsto e)\}$ and $\operatorname{exit}(\mathcal{E}) \triangleq\{e \in$ $E \mid l(e)=\delta\}$.

$\mathcal{E} \llbracket \rrbracket$ is defined recursively in Definition 21 . We suppose there is an infinite universe $E_{U}$ of events. In the rest of this section let $\mathcal{E} \llbracket B_{i} \rrbracket=\mathcal{E}_{i}=\left(E_{i}, \rightsquigarrow_{i}, \mapsto_{i}, l_{i}\right)$, for $i=1,2$ with $E_{1} \cap E_{2}=\varnothing$. (If $E_{1} \cap E_{2} \neq \varnothing$ then a suitable event renaming can be applied extended to $\rightsquigarrow, \mapsto$ and $l$.)

The semantics of $\mathbf{0}$ and $\sqrt{ }$ is self-explanatory. In $\mathcal{E} \llbracket a ; B_{1} \rrbracket$ a bundle is introduced from the new event $e_{a}$ (labelled $a$ ) to all initial events in $\mathcal{E}_{1}$ as $e_{a}$ causally precedes these events. $\mathcal{E} \llbracket B_{1}+B_{2} \rrbracket$ is equal to $\mathcal{E}_{1} \cup \mathcal{E}_{2}$ extended with mutual conflicts between all initial events of $\mathcal{E}_{1}$ and $\mathcal{E}_{2}$ such that in the resulting structure only either $B_{1}$ or $B_{2}$ can happen.

$\mathcal{E} \llbracket B_{1} \backslash G \rrbracket$ is identical to $\mathcal{E}_{1}$ except that events labelled with a label in $G$ are now labelled with $\tau$ turning those events into internal ones. $\mathcal{E} \llbracket B_{1}[H] \rrbracket$ is defined similarly where events are relabelled according to $H$ (o denotes usual function composition).

$\mathcal{E} \llbracket B_{1} \gg B_{2} \rrbracket$ is equal to $\mathcal{E}_{1} \cup \mathcal{E}_{2}$ where bundles are introduced from the successful termination events of $\mathcal{E}_{1}$ to the initial events of $\mathcal{E}_{2}$. (To create bundles, mutual 
conflicts are introduced between the successful termination events of $\mathcal{E}_{1}$.) This corresponds with the fact that these initial events can only occur if $B_{1}$ has successfully terminated. The successful termination events of $\mathcal{E}_{1}$ are relabelled into internal events.

$\mathcal{E} \llbracket B_{1} \llbracket>B_{2} \rrbracket$ is equal to $\mathcal{E}_{1} \cup \mathcal{E}_{2}$ extended with some additional asymmetric conflicts. First, each event in $\mathcal{E}_{1}$ may be disabled by an initial event of $\mathcal{E}_{2}$. This models that $B_{1}$ is disrupted once an initial event of $B_{2}$ happens. In addition, after the occurrence of a successful termination event in $\mathcal{E}_{1}$ no initial event of $\mathcal{E}_{2}$ can happen anymore.

We finally consider parallel composition. The events of $\mathcal{E} \llbracket B_{1} \|_{G} B_{2} \rrbracket$ are constructed in the following way: an event $e$ of $\mathcal{E}_{1}$ or $\mathcal{E}_{2}$ that does not need to synchronize is paired with the auxiliary symbol $*$, and an event which is labelled with an action in $G^{\delta}$ is paired with all events (if any) in the other process that are equally labelled. Thus events are pairs of events of $\mathcal{E}_{1}$ and $\mathcal{E}_{2}$, or with one component equal to *. Two events are now put in conflict if any of their components are in conflict, or if different events have a common component different from $*$ (such events appear if two or more events in one process synchronize with the same event in the other process). A bundle is introduced such that if we take the projection on the $i$-th component $(i=1,2)$ of all events in the bundle we obtain a bundle in $\mathcal{E} \llbracket B_{i} \rrbracket$.

For $G \subseteq$ Act, $E_{i}^{s} \triangleq\left\{e \in E_{i} \mid l_{i}(e) \in G^{\delta}\right\}$ is the set of synchronization events and $E_{i}^{f} \triangleq E_{i} \backslash E_{i}^{s}$ the set of non-synchronizing events.

Definition 21. $\mathcal{E} \llbracket \rrbracket:$ PA $\longrightarrow$ EBES is defined as follows:

$$
\begin{aligned}
& \mathcal{E} \llbracket \mathbf{0} \rrbracket \triangleq(\varnothing, \varnothing, \varnothing, \varnothing) \\
& \mathcal{E} \llbracket \sqrt{ } \rrbracket \triangleq\left(\left\{e_{\delta}\right\}, \varnothing, \varnothing,\left\{\left(e_{\delta}, \delta\right)\right\}\right) \text { for some } e_{\delta} \in E_{U} \\
& \mathcal{E} \llbracket a ; B_{1} \rrbracket \triangleq\left(E, \rightsquigarrow 1, \mapsto, l_{1} \cup\left\{\left(e_{a}, a\right)\right\}\right) \text { where } \\
& E=E_{1} \cup\left\{e_{a}\right\} \text { for some } e_{a} \in E_{U} \backslash E_{1} \\
& \mapsto=\mapsto_{1} \cup\left(\left\{\left\{e_{a}\right\}\right\} \times \operatorname{init}\left(\mathcal{E}_{1}\right)\right) \\
& \mathcal{E} \llbracket B_{1}+B_{2} \rrbracket \triangleq\left(E_{1} \cup E_{2}, \rightsquigarrow, \mapsto_{1} \cup \mapsto_{2}, l_{1} \cup l_{2}\right) \text { where } \\
& \rightsquigarrow=\rightsquigarrow_{1} \cup \rightsquigarrow_{2} \cup\left(\operatorname{init}\left(\mathcal{E}_{1}\right) \times \operatorname{init}\left(\mathcal{E}_{2}\right)\right) \cup\left(\operatorname{init}\left(\mathcal{E}_{2}\right) \times \operatorname{init}\left(\mathcal{E}_{1}\right)\right) \\
& \mathcal{E} \llbracket B_{1} \backslash G \rrbracket \triangleq\left(E_{1}, \rightsquigarrow_{1}, \mapsto_{1}, l\right) \text { where } \\
& \left(l_{1}(e) \in G \Rightarrow l(e)=\tau\right) \wedge\left(l_{1}(e) \notin G \Rightarrow l(e)=l_{1}(e)\right) \\
& \mathcal{E} \llbracket B_{1}[H] \rrbracket \triangleq\left(E_{1}, \rightsquigarrow_{1}, \mapsto_{1}, H \circ l_{1}\right) \\
& \mathcal{E} \llbracket B_{1} \gg B_{2} \rrbracket \triangleq\left(E_{1} \cup E_{2}, \rightsquigarrow, \mapsto, l\right) \text { where } \\
& \rightsquigarrow=\rightsquigarrow_{1} \cup \rightsquigarrow_{2} \cup\left\{\left(e, e^{\prime}\right) \mid e, e^{\prime} \in \operatorname{exit}\left(\mathcal{E}_{1}\right) \wedge e \neq e^{\prime}\right\} \\
& \mapsto=\mapsto_{1} \cup \mapsto_{2} \cup\left(\left\{\operatorname{exit}\left(\mathcal{E}_{1}\right)\right\} \times \operatorname{init}\left(\mathcal{E}_{2}\right)\right) \\
& l=\left(\left(l_{1} \cup l_{2}\right) \backslash\left(\operatorname{exit}\left(\mathcal{E}_{1}\right) \times\{\delta\}\right)\right) \cup\left(\operatorname{exit}\left(\mathcal{E}_{1}\right) \times\{\tau\}\right) \\
& \mathcal{E} \llbracket B_{1} \llbracket>B_{2} \rrbracket \triangleq\left(E_{1} \cup E_{2}, \rightsquigarrow, \mapsto_{1} \cup \mapsto_{2}, l_{1} \cup l_{2}\right) \text { where } \\
& \rightsquigarrow=\rightsquigarrow_{1} \cup \rightsquigarrow_{2} \cup\left(E_{1} \times \operatorname{init}\left(\mathcal{E}_{2}\right)\right) \cup\left(\operatorname{init}\left(\mathcal{E}_{2}\right) \times \operatorname{exit}\left(\mathcal{E}_{1}\right)\right) \\
& \mathcal{E} \llbracket B_{1} \|_{G} B_{2} \rrbracket \triangleq(E, \rightsquigarrow, \mapsto, l) \text { where } \\
& E=\left(E_{1}^{f} \times\{*\}\right) \cup\left(\{*\} \times E_{2}^{f}\right) \cup \\
& \left\{\left(e_{1}, e_{2}\right) \in E_{1}^{s} \times E_{2}^{s} \mid l_{1}\left(e_{1}\right)=l_{2}\left(e_{2}\right)\right\}
\end{aligned}
$$




$$
\begin{aligned}
\left(e_{1}, e_{2}\right) \rightsquigarrow\left(e_{1}^{\prime}, e_{2}^{\prime}\right) \Leftrightarrow & \left(e_{1} \rightsquigarrow_{1} e_{1}^{\prime}\right) \vee\left(e_{2} \rightsquigarrow_{2} e_{2}^{\prime}\right) \vee \\
& \left(e_{1}=e_{1}^{\prime} \neq * \wedge e_{2} \neq e_{2}^{\prime}\right) \vee\left(e_{2}=e_{2}^{\prime} \neq * \wedge e_{1} \neq e_{1}^{\prime}\right) \\
X \mapsto\left(e_{1}, e_{2}\right) \Leftrightarrow \quad\left(\exists X_{1} \subseteq E_{1}: X_{1} \mapsto_{1} e_{1} \wedge X=\left\{\left(e, e^{\prime}\right) \in E \mid e \in X_{1}\right\}\right) & \vee\left(\exists X_{2} \subseteq E_{2}: X_{2} \mapsto_{2} e_{2} \wedge X=\left\{\left(e, e^{\prime}\right) \in E \mid e^{\prime} \in X_{2}\right\}\right) \\
l\left(\left(e_{1}, e_{2}\right)\right)= & \text { if } e_{1}=* \text { then } l_{2}\left(e_{2}\right) \text { else } l_{1}\left(e_{1}\right) .
\end{aligned}
$$

In this paper we use a slightly different version of $\mathcal{E} \llbracket \rrbracket$, denoted $\mathcal{E}^{\prime} \llbracket \rrbracket$. The only difference with $\mathcal{E} \llbracket \rrbracket$ is that for action-prefix $\mathcal{E}^{\prime} \llbracket \rrbracket$ introduces not only bundles from $e_{a}$ to the initial events of $\Gamma_{1}$ (asabove), but to all events in $\Gamma_{1}$. Similarly, for sequential composition $\mathcal{E}^{\prime} \llbracket \rrbracket$ introduces bundles from the successful termination events of $\Gamma_{1}$ to all events in $\Gamma_{2}$. These additional bundles do not pose any problems:

Proposition 22. $\forall B \in \mathrm{PA}: T(\mathcal{E} \llbracket B \rrbracket)=T\left(\mathcal{E}^{\prime} \llbracket B \rrbracket\right)$.

\section{References}

1. L. Aceto and D. Murphy. On the ill-timed but well-caused. In E. Best, editor, Concur' 93, LNCS 715, pages 97-111. Springer-Verlag, 1993.

2. L. Aceto and D. Murphy. Timing and causality in process algebra. Acta Informatica, 1996. (to appear).

3. R. Alur and D.L. Dill. A theory of timed automata. Theoretical Computer Science, 126:183-235, 1994.

4. J.C.M. Baeten and J.A. Bergstra. Real time process algebra. Formal Aspects of Computing, 3(2):142-188, 1991.

5. T. Bolognesi and E. Brinksma. Introduction to the ISO specification language LOTOS. Computer Networks and ISDN Systems, 14:25-59, 1987.

6. G. Boudol and I. Castellani. Flow models of distributed computations: three equivalent semantics for CCS. Information \&S Computation, 114: 247-314, 1994.

7. E Brinksma, J.-P. Katoen, R. Langerak, and D. Latella. Performance analysis and true concurrency semantics. In T. Rus and C. Rattray, editors, Theories and Experiences for Real-Time System Development, volume 2 of AMAST Series in Computing, chapter 12, pages 309-337. World Scientific, 1994.

8. R.T. Casley, R.F. Crew, J. Meseguer, and V.R. Pratt. Temporal structures. Mathematical Structures in Computer Science, 1(2):179-213, 1991.

9. J.W. de Bakker, W.-P. de Roever, and G. Rozenberg, editors. Linear Time, Branching Time and Partial Order in Logics and Models for Concurrency, LNCS 354. SpringerVerlag, 1989.

10. C. Fidge. A constraint-oriented real-time process calculus. In M. Diaz and R. Groz, editors, FORTE'92 - Fifth International Conference on Formal Description Techniques, volume C-10 of IFIP Transactions, pages 363-378. North-Holland, 1993.

11. R. Gorrieri, M. Roccetti, and E. Stancampiano. A theory of processes with durational actions. Theoretical Computer Science, 140:73-94, 1995.

12. J. Gunawardena. A dynamic approach to timed behaviour. In B. Jonsson and J. Parrow, editors, Concur' 94: Concurrency Theory, LNCS 836, pages 178-193. Springer-Verlag, 1994. 
13. W. Janssen, M. Poel, Q. Wu, and J. Zwiers. Layering of real-time distributed processes. In H. Langmaack, W.-P. de Roever, and J. Vytopil, Formal Techniques in Real-Time and Fault-Tolerant Systems, LNCS 863, pages 393-417. Springer-Verlag, 1994.

14. J.-P. Katoen. Quantitative and Qualitative Extensions of Event Structures. PhD thesis, University of Twente, 1996.

15. J.-P. Katoen, R. Langerak, and D. Latella. Modelling systems by probabilistic process algebra: An event structures approach. In R.L. Tenney, P.D. Amer, and M.Ü. Uyar, editors, Formal Description Techniques VI, volume C-22 of IFIP Transactions, pages 253-268. North-Holland, 1994.

16. J.-P. Katoen, D. Latella, R. Langerak, E. Brinksma, and T. Bolognesi. A consistent causality-based and interleaved view on a timed process algebra including timeouts. In A. Cornell and D. Ionescu, Proceedings 3rd Amast Workshop on Real-Time System Development, 1996.

17. R. Langerak. Transformations and Semantics for LOTOS. PhD thesis, University of Twente, 1992

18. A. Maggiolo-Schettini and J. Winkowski. Towards an algebra for timed behaviours. Theoretical Computer Science, 103:335-363, 1992.

19. A. Mazurkiewicz. Basic notions of trace theory. In de Bakker et al. [9], pages 285-363.

20. D. Murphy. Time and duration in noninterleaving concurrency. Fundamenta Informaticae, 19:403-416, 1993.

21. X. Nicollin and J. Sifakis. An overview and synthesis on timed process algebras. In J.W. de Bakker, C. Huizing, W.-P. de Roever, and G. Rozenberg, editors, Real-Time: Theory in Practice, LNCS 600, pages 526-548. Springer-Verlag, 1992.

22. G.M. Pinna and A. Poigné. On the nature of events: another perspective in concurrency. Theoretical Computer Science, 138(2):425-454, 1995.

23. G.D. Plotkin. A structural approach to operational semantics. Technical Report DAIMI FN-19, Computer Science Department, Aarhus University, 1981.

24. V.R. Pratt. Modeling concurrency with partial orders. International Journal of Parallel Programming, 15(1):33-71, 1986.

25. J. Quemada, D. de Frutos, and A. Azcorra. TIC: A TImed Calculus. Formal Aspects of Computing, 5:224-252, 1993.

26. A. Rensink. Methodological aspects of action refinement. In E.-R. Olderog, editor, Programming Concepts, Methods and Calculi, volume A-56 of IFIP Transactions, pages 227-246. North-Holland, 1994.

27. D.A. Schmidt. Denotational Semantics: a methodology for language development. Allyn and Bacon, 1986.

28. C.A. Vissers. FDTs for open distributed systems, a retrospective and a prospective view. In L. Logrippo, R.L. Probert, and H. Ural, editors, Protocol Specification, Testing and Verification X, pages 341-362. North-Holland, 1990.

29. G. Winskel. An introduction to event structures. In de Bakker et al. [9], pages 364397.

30. J.J. Zic. Time-constrained buffer specifications in CSP+T and Timed CSP. ACM Transactions on Programming Languages and Systems, 16(6):1661-1674, 1994.

This article was processed using the $\mathrm{IAT}_{\mathrm{E}} \mathrm{X}$ macro package with LLNCS style 